\title{
Dissolution of nucleons in giant nuclei
}

\author{
David Vasak, Walter Greiner, and Ludwig Neise \\ Institut für Theoretische Physik der Johann Wolfgang Goethe-Universität, 6000 Frankfurt am Main, Federal Republic of Germany \\ and Nuclear Science Division, Lawrence Berkeley Laboratory, University of California, Berkeley, California 94720
}

(Received 4 November 1985)

\begin{abstract}
We discuss the possibility that nuclei with very large baryon numbers can exist in the form of large quark blobs in their ground states. A calculation based on the picture of quark bags shows that, in principle, the appearance of such exotic nuclear states in present laboratory experiments cannot be excluded. Some speculations in connection with the recently observed anomalous positron production in heavy-ion experiments are presented.
\end{abstract}

\section{INTRODUCTION}

On the fundamental level of quantum chromodynamics (QCD), the nucleon-nucleon interaction appears to be an effective description of forces acting between the truly elementary particles, the quarks and gluons. The hadrons, and among them also the nucleons, are composite objects containing small numbers of quarks trapped within a limited region of space. Due to the complex algebraic structure of the underlying symmetry group, $\mathrm{SU}(3)_{c}$, which, for example, has the consequence that the eight mediators of this interaction, the gluons, interact with each other, even rather elementary calculations turn out to be possible only with the aid of large computers.

Model builders have, however, anticipated the results of future exact calculations, and developed the so-called "bag" picture of hadrons." In this approach the strong interaction is thought to be split into two parts. The first one, which describes the nonperturbative and non-Abelian part responsible for the vacuum structure and the confinement of quarks, cannot be calculated explicitly and is built in "by hand." Excitations of the ground state are bubblelike inhomogeneities in the vacuum condensate, filled with quarks which can be also regarded as local minima in a kind of self-consistent potential analogous to the effective pairing potential known from the LandauGinsburg theory of superconducting materials. ${ }^{2}$ Hadrons are nothing other than such holes in the vacuum medium dug by the quarks themselves. First order perturbation theory (one-gluon exchange) turns out to be sufficient for the residual part of the interaction, when baryons and, to some degree also mesons, with a small number of quarks are considered.

Models based on this semiphenomenological point of view exist in various degrees of sophistication (for example, the MIT bag, ${ }^{3}$ the soliton bag, ${ }^{4}$ the chiral bag, ${ }^{5}$ the hybrid bag, ${ }^{6}$ etc. ${ }^{1}$ ). In all of them the confining effect of the surrounding vacuum is achieved through a substantial increase of the quark mass across the boundary of the bubble.

Conventionally, atomic nuclei are considered to be composed of nucleons, treated as its fundamental (pointlike) components which do not change their properties when embedded in nuclear matter and which interact via some quite involved and empirically determined interaction. This point of view has lead to substantial success in explaining low energy data and the static properties of atomic nuclei. Recent experiments, performed by the European Muon Collaboration (EMC), revealing the high momentum (i.e., small distance) structure of nuclear matter ${ }^{7}$ force us, however, to modify this point of view, since the internal structure of nucleons embedded in nuclei seems to be altered. The degree of modification of the form factor even increases with the size of the surrounding nucleus.

In the framework of the bag models, the abovementioned effect on the nucleon structure can be well understood. ${ }^{8}$ Although the quarks feel "uncomfortable" outside the bag, when the exterior is a simple homogeneous condensate, i.e., when the hadron (nucleon) is isolated, they may leak out, if the vacuum becomes structured in its vicinity due to the presence of other hadrons. Moreover, since the condensate itself responds to the quark structure in a self-consistent way, the inhomogeneities themselves should change, too. The probability for a colored quark to be outside the free nucleon thus increases, i.e., the confinement radius becomes effectively larger, the nucleons overlap, and color conductivity sets in. ${ }^{9}$ With increasing delocalization of the quarks, a color band structure can develop, resembling band effects known from the elementary physics of conducting crystals. Corresponding calculations for periodic bag structures have been done in the framework of the hybrid bag model ${ }^{10}$ and of the MIT bag model. ${ }^{11}$

In this paper we do not wish to add another piece of work to the already existing vast amount of literature on the explanation of the EMC effect, ${ }^{8}$ but take the picture discussed above seriously and extrapolate it to very large nuclei: If the color conductivity within nuclei increases with their increasing size, and if this increase is connected with a continuous depletion of the inhomogeneities in the "condensate field" (i.e., the $\sigma$ field in the language of the soliton-bag model), then in giant nuclei with nucleon number $\boldsymbol{A}$ of the order of several hundreds this field should vanish completely in the interior, turning the giant nucleus into a cold quark gas. These objects, which we 
call giant quark nuclei (GQN), are investigated here. ${ }^{12}$ The question arises, of course, whether the perturbative treatment of the residual quark-quark interaction is applicable also when the number of quarks and the size of the "hadron" are very large. Here we simply neglect the residual interactions. One can argue against this point of view, but lacking any conclusive prescription how to handle the residual interactions, our calculation can at least serve as a basis for future improvements.

The transition of ordinary nuclear matter into a color conductor, which we have in mind here, seems to be rather analogous to similar phenomena observed in solid state physics, when clusters of atoms are studied with respect to the development of collective properties like conductivity or superconductivity. There it might be that such properties do not appear, if the number of participating atoms is relatively small $\left(10^{2}, 10^{3}\right)$, but that they set in beyond a certain cluster size. ${ }^{13}$

There is yet another motivation for our investigation on giant quark nuclei, emerging from the very exciting studies of the decay of the electron-positron vacuum in supercritical electric fields generated by two very heavy ions in collisions just above the Coulomb barrier. ${ }^{14}$ Many experimental hints point towards the discovery of positrons spontaneously produced in this fundamental process and also towards the existence of giant nuclei. The intimate link between these two concepts is due to the appearance of a main and several secondary positron resonance lines at various energies and in different "windows," which possibly indicate the existence of surprisingly long-lived nuclear objects. Even though phenomenological studies of unified nuclear systems show that nuclear molecular states are not unlikely, ${ }^{15}$ giant nuclei seem to be difficult to justify on the basis of conventional nuclear physics ${ }^{16}$ or even in meson field theory. ${ }^{17}$ Nevertheless, the strange scaling property of the experimentally observed and so far principally investigated positron peaks definitely requires a new type of nuclear structure.

The paper is organized in the following way: In Sec. II we introduce a simple model to estimate the binding energy per nucleon of giant quark nuclei in the framework of the MIT bag approach, and compare the results with those obtained from the extrapolation of the empirical Bethe-Weizsäcker formula fitted to known nuclei. In Sec. III we discuss the physical content of this calculation and its consequences in the context of the bulk of known data. Finally, speculations about experimental consequences seem appropriate.

\section{MODEL FOR GIANT QUARK NUCLEI}

We consider now a nucleus with the mass number $A$, in which quarks are not clustered into nucleons, but move freely throughout the interior of the whole nucleus. The confining force keeping the quarks from leaving the nucleus can be understood as the action of the surrounding "true" QCD vacuum in which colored objects may not propagate unless they are combined into color singlets. Such nuclei (or hadrons in general) form "bubbles" in the "true vacuum" filled with quarks. These bubbles correspond to local collective vacuum excitations of the collec- tive condensate field. The amount of energy which has to be invested to create such an excitation is proportional to the volume $V$ of the bubble. The corresponding energy density $B$ is a universal constant, which has to be fitted to experiments, as long as we are not able to connect it with the QCD scale parameter by exact calculations (see, however, Ref. 18). In the MIT approach ${ }^{3}$ such bubbles are described as cavities with sharp boundaries, in which the quarks (and eventually also gluons) move freely, obeying the free equations of motion (we neglect here, as discussed above, the residual interactions):

$$
i \gamma_{\mu} \partial^{\mu} \psi(x)=0
$$

where $\psi$ is a four-component spinor; cf. Eq. (30). Our units are such that $\hbar=c=1$.

On the surface, however, their wave functions have to satisfy the boundary conditions ( $n^{\mu}$ is the surface normal vector)

$$
\begin{aligned}
& i \gamma_{\mu} n^{\mu} \psi=\psi \text { on the surface, } \\
& -\frac{1}{2} n^{\mu} \partial_{\mu} \bar{\psi} \psi=B \text { on the surface, }
\end{aligned}
$$

which mimic the infinite discontinuity of the quark mass across the boundary. This leads to a discrete single particle energy (and momentum) spectrum, displayed in Table I for massless quarks in the convenient dimensionless form

$$
\omega_{\kappa n}=R E_{\kappa n},
$$

where $E_{\kappa n}$ is the eigenfrequency of the quark level with the Dirac quantum number $\kappa$ and the principal quantum number $n$, and $R$ is the radius of the spherical cavity.

Adding a nucleon into the bag corresponds to the addition of two up and one down quark for a proton, and of one up and two down quarks for a neutron. Whereas the color interaction responsible for the confinement does not depend on flavor, the electromagnetic charge becomes important when considering the effects of the Coulomb energy. This will be done schematically using the following approximation. Suppose the nucleus is a homogeneously charged sphere of radius $R$. The Coulomb energy of such a sphere (total charge $\mathrm{Ze}$ ) is

$$
E_{c}^{\prime}=3 \alpha Z^{2} / 5 R \text {, }
$$

where $\alpha=e^{2} / 4 \pi=1 / 137$ is the fine-structure constant. Now the up and down quarks (numbers $Z_{u}$ and $Z_{d}$, respectively) carry the fractional electric charges $2 e / 3$ and $-e / 3$, such that the required total charge of our nucleus is

$$
Z=\frac{2}{3} Z_{u}-\frac{1}{3} Z_{d}
$$

and the number of neutrons $A-Z$. Because

$$
\begin{aligned}
& Z_{u}+Z_{d}=3 A, \\
& Z_{u}=A+Z, \\
& Z_{d}=2 A-Z .
\end{aligned}
$$

After inserting (3) into (2) we obtain the Coulomb energy

$$
E_{c}^{\prime}=3 \alpha / 5 R\left(2 Z_{u} / 3-Z_{d} / 3\right)^{2}
$$


of a homogeneously charged sphere consisting of $Z_{u}$ up and $Z_{d}$ down quarks. In this expression, however, the Coulombic self-interaction of each quark is also counted. This can be renormalized away by replacing

$$
Z_{i}^{2} \rightarrow Z_{i}\left(Z_{i}-1\right) \quad i=u, d
$$

in Eq. (6), thus yielding

$$
\begin{aligned}
E_{c} & =\alpha / 15 R\left[4 Z_{u}\left(Z_{u}-1\right)+Z_{d}\left(Z_{d}-1\right)-4 Z_{u} Z_{d}\right] \\
& \equiv \omega_{c} / R
\end{aligned}
$$

Insertion of the Coulomb energy makes the proton heavier than the neutron. We can remedy this by realizing that the mass of the down quark is larger than that of the up quark. A reasonable assumption, which is consistent with standard bag calculations, is $m_{u}=0, m_{d}=5 \mathrm{MeV}$. This small mass leads to a modification of the boundary condition for the quark wave functions. ${ }^{3}$ If we expand the corresponding equation around $\mu=0$, in first order of $\mu=m_{d} R$ the arising correction of the momentum eigenvalue is estimated to be

$$
\omega_{\kappa n}(\mu)=\omega_{\kappa n}(0)+\frac{1}{2} \mu /\left[\omega_{\kappa n}(0)+\kappa\right],
$$

and for the energy we obtain as usual

$$
\epsilon_{\kappa n}(\mu)=\left[\omega_{\kappa n}^{2}(\mu)+\mu^{2}\right]^{1 / 2} .
$$

With these considerations the total energy of the bag is simply ${ }^{3}$

$$
\begin{aligned}
E_{\mathrm{tot}}(A, Z, R)= & \sum \epsilon_{\kappa n} / R+E_{c}\left[Z_{u}(A, Z), Z_{d}(A, Z)\right] \\
& +4 \pi B R^{3} / 3 .
\end{aligned}
$$

The sum in the first term corresponds to the kinetic energy of the quarks and runs over all occupied modes, the second term is the renormalized Coulomb energy (8), and the last one the condensation or volume energy.

To be definite we employ in the following the "orthodox" value 19 of $B=(145 \mathrm{MeV})^{4}$, and shall discuss the great dependence of our results on the bag constant in the

\begin{tabular}{|c|c|c|c|c|c|}
\hline$\omega_{n}$ & $\kappa$ & & $\omega_{\kappa n}$ & $\kappa$ & \\
\hline 2.04278694273 & -1 & 6 & 12.33348062758 & 5 & 822 \\
\hline 3.20391876726 & -2 & 18 & 12.70944981993 & -4 & 846 \\
\hline 3.81153864777 & 1 & 24 & 12.90161883497 & -11 & 912 \\
\hline 4.32730291250 & -3 & 42 & 12.96372863983 & -7 & 954 \\
\hline 5.12311062410 & 2 & 54 & 13.00886854595 & 3 & 972 \\
\hline 5.39601611785 & -1 & 60 & 13.19690694178 & -2 & 984 \\
\hline 5.42952237092 & -4 & 84 & 13.31559357684 & 1 & 990 \\
\hline 6.37113691533 & 3 & 102 & 13.35096234860 & 9 & 1044 \\
\hline 6.51788754767 & -5 & 132 & 13.58486809101 & 6 & 1080 \\
\hline 6.75780501185 & -2 & 144 & 13.95153160392 & -12 & 1152 \\
\hline 7.00203329571 & 1 & 150 & 14.01249543641 & -5 & 1182 \\
\hline 7.58129857678 & 4 & 174 & 14.14515954635 & -8 & 1230 \\
\hline 7.59634581701 & -6 & 210 & 14.36722103420 & 4 & 1254 \\
\hline 8.05957827856 & -3 & 228 & 14.47248193811 & 10 & 1314 \\
\hline 8.40758707164 & 2 & 240 & 14.60960858443 & -3 & 1332 \\
\hline 8.57755878461 & -1 & 246 & 14.78940655650 & 2 & 1344 \\
\hline 8.66730055378 & -7 & 288 & 14.81738110856 & 7 & 1386 \\
\hline 8.76571372299 & 5 & 318 & 14.88782748573 & -1 & 1392 \\
\hline 9.32191544672 & -4 & 342 & 14.99890082782 & -13 & 1470 \\
\hline 9.73233713563 & -8 & 390 & 15.29173705254 & -6 & 1506 \\
\hline 9.75354968367 & 3 & 408 & 15.31495554700 & -9 & 1560 \\
\hline 9.93122588787 & 6 & 444 & 15.58709297961 & 11 & 1626 \\
\hline 10.00419347084 & -2 & 456 & 15.69559853618 & 5 & 1656 \\
\hline 10.16332073509 & 1 & 462 & 15.98579945042 & -4 & 1680 \\
\hline 10.55591883243 & -5 & 492 & 16.03430698797 & 8 & 1728 \\
\hline 10.79256324954 & -9 & 546 & 16.04402993356 & -14 & 1812 \\
\hline 11.05862603181 & 4 & 570 & 16.21798518269 & 3 & 1830 \\
\hline 11.08213825437 & 7 & 612 & 16.36909138309 & -2 & 1842 \\
\hline 11.37644149760 & -3 & 630 & 16.46389560006 & 1 & 1848 \\
\hline 11.61203022487 & 2 & 642 & 16.47485855825 & -10 & 1908 \\
\hline 11.73650395934 & -1 & 648 & 16.55150457940 & -7 & 1950 \\
\hline 11.76836247041 & -6 & 684 & 16.69571662752 & 12 & 2022 \\
\hline 11.84878712545 & -10 & 744 & 16.99980620134 & 6 & 2058 \\
\hline 12.22135972278 & 8 & 792 & 17.08716811425 & -15 & 2148 \\
\hline
\end{tabular}
next section. To take also into account the correction for the spurious collective motion of the center of mass of the quark distribution relative to the cavity center, we replace $^{6}$

TABLE I. Cavity modes $\omega_{\kappa n} \leq 17$ in ascending order. The Dirac quantum numbers $\kappa$ are also displayed as well as the number of quarks with a particular flavor, filling all levels up to the given one. 


$$
\sum \epsilon_{\kappa n} \rightarrow\left[\left(\sum \epsilon_{\kappa n}\right]^{2}-\sum \omega_{\kappa n}^{2}\right]^{1 / 2}
$$

thus subtracting the contribution of the average total momentum from the total squared quark kinetic energy (this corresponds to the value $Z_{0}=1.12$ for the constant in the conventional correction term ${ }^{2}$ for the nucleon). Note that for increasing number of occupied modes

$$
\left(\sum \omega_{\kappa n}\right)^{2} \gg \sum \omega_{\kappa n}^{2}
$$

and the c.m. correction becomes negligible for $G Q N$ as does the zero-point energy, ${ }^{1,2}$ which is of the order $1 / R$. While the former is built into our numerical code, we omit the latter for convenience.
A giant quark bag in its ground state will adjust the numbers of up and down quarks (for a given nucleon number $A$ ) and the radius $R$ such that the pressure of the quarks on the bag surface is balanced by the pressure exerted by the surrounding vacuum, or, equivalently, ${ }^{1}$ that the total energy $E_{\text {tot }}$ is minimal:

$$
M_{\mathrm{GQN}}(A)=\min _{\{Z, R\}} E_{\mathrm{tot}}(A, Z, R) .
$$

This minimization will be performed in two steps. From

$$
\partial / \partial R E_{\text {tot }} \mid R=R_{0}=0
$$

we obtain immediately, if we neglect for a moment the finite mass of the down quark and recognize the $R$ independence of both $\omega_{\kappa n}$ and $\omega_{c}$,

$$
\begin{aligned}
& R_{0}(A, Z)=\left\{\left\{\left[\left(\sum \omega_{\kappa n}\right]^{2}-\sum \omega_{\kappa n}^{2}\right]^{1 / 2}+\omega_{c}(Z)\right\} / 4 \pi B\right]^{1 / 4}, \\
& E_{\mathrm{tot}}^{0}(A, Z)=16 \pi B R_{0}^{3}(A, Z) / 3
\end{aligned}
$$

for the mass of the giant bag. From (14) and (15) it is clear that $R_{0}$ and consequently also $M_{\mathrm{GQN}}$ will be minimal, if $Z$ is adjusted to minimize the nominator in (14). To develop a convenient algorithm for the filling scheme including the effects of the Coulomb interaction we first list below two recurrence relations:

$$
\begin{aligned}
\Delta_{u} E_{c}\left(Z_{u}, Z_{d}\right) & \equiv E_{c}\left(Z_{u}+1, Z_{d}\right)-E_{c}\left(Z_{u}, Z_{d}\right) \\
& =4 \alpha / 15 R\left(2 Z_{u}-Z_{d}\right) \\
& \equiv \Delta_{u} \omega_{c}\left(Z_{u}, Z_{d}\right) / R \\
\Delta_{d} E_{c}\left(Z_{u}, Z_{d}\right) & \equiv E_{c}\left(Z_{u}, Z_{d}+1\right)-E_{c}\left(Z_{u}, Z_{d}\right) \\
& =-2 \alpha / 15 R\left(2 Z_{u}-Z_{d}\right) \\
& \equiv \Delta_{d} \omega_{c}\left(Z_{u}, Z_{d}\right) / R
\end{aligned}
$$

As an example of their application we calculate the masses of the first four nuclei with $A=1,2,3,4$. For this purpose only the two lowest quark levels have to be taken into consideration. The degeneracy of each level is $2 j+1=2|\kappa|$ for angular momentum, 3 for color, and 2 for isospin. The first $\left(1 s_{1 / 2}\right)$ level has the eigenfrequency

$$
\omega_{-1,1}=2.043
$$

and a total degeneracy of 12 . The quark content of the $A=1$ bag, i.e., the "nucleon," is ( $u u d)$ if the stable lowest energy configuration is a proton, and $(u d d)$ if the stable configuration is a neutron. ${ }^{20}$ In both cases, however, one up and down quark are present. We therefore obtain first

$$
\begin{aligned}
R E_{u d} & =\omega_{-1,1}+\Delta_{u} \omega_{c}(0,0)+\omega_{-1,1}+\Delta_{d} \omega_{c}(1,0) \\
& =4.086-4 \alpha / 15
\end{aligned}
$$

and then for the proton configuration $(u d u)$

$$
\begin{aligned}
R E_{u d u} & =R E_{u d}+\omega_{-1,1}+\Delta_{u} \omega_{c}(1,1) \\
& =6.129-4 \alpha / 15+4 \alpha / 15=6.129,
\end{aligned}
$$

and for the neutron configuration ( $u d d)$

$$
\begin{aligned}
R E_{u d d} & =R E_{u d}+\omega_{-1,1}+\Delta_{d} \omega_{c}(1,1) \\
& =6.129-4 \alpha / 15-2 \alpha / 15=6.129-2 \alpha / 5 .
\end{aligned}
$$

Obviously, the neutron has lower mass than the proton (if the nucleon radius is $1 \mathrm{fm}$ the corresponding energy difference is $\sim 0.58 \mathrm{MeV}$ ) and is a stable particle in this approach with the down-quark mass kept at zero. Let us now proceed with constructing the next heavier nucleus. First we add again another $u d$ combination to the neutron to obtain

$$
\begin{aligned}
R E_{u d d u d}= & R E_{u d d}+2 \omega_{-1,1}+\Delta_{u} \omega_{c}(1,2) \\
& +\Delta_{d} \omega_{c}(2,2) \\
= & 10.205-2 \alpha / 5+0-4 \alpha / 15 \\
= & 10.205-2 \alpha / 3,
\end{aligned}
$$

and by inspection of the neutron-proton configuration

$$
\begin{aligned}
R E_{u d d u d u} & =12.248-2 \alpha / 3+\Delta_{u} \omega_{c}(2,3) \\
& =12.248-2 \alpha / 5,
\end{aligned}
$$

and of the dineutron configuration

$$
\begin{aligned}
R E_{u d d u d d} & =12.248-2 \alpha / 3+\Delta_{d} \omega_{c}(2,3) \\
& =12.248-4 \alpha / 5,
\end{aligned}
$$

the dineutron (21b) turns out to be the next stable configuration. If we add another three quarks we find the trineutron to again fulfill the condition of minimal ener- 
gy. Since now the Pauli principle forbids us to add another down quark into the $1 s_{1 / 2}$ shell, and the next eigenmode

$$
\omega_{2,1}=3.204
$$

is appreciably higher, the $A=4$ nucleus will contain at least one proton, because the addition of an upper quark into the $1 s_{1 / 2}$ shell is energetically favorable over adding a down quark into the $1 p_{3 / 2}$ shell. This action of the Pauli principle has apparently similar consequences as the "symmetry energy" in the conventional language of the liquid-drop theory.

For each case, i.e., for $A=1,2, \ldots$, we thus chose the charge $Z=Z_{\min }$, which corresponds to minimum energy, and calculate the equilibrium radius $R_{0}(A)$ from Eq. (14) to obtain the total bag mass

$$
M_{\text {tot }}(A)=E_{\text {tot }}^{0}\left(A, Z=Z_{\min }\right) .
$$

The equilibrium radius is plotted in Fig. 1 as function of the mass number $A$ in the form

$$
r_{0}(A)=R_{0}(A) / A^{1 / 3} \text {. }
$$

The dotted curve is $r_{0}(A)$ calculated for bags containing only free quarks (no Coulomb interaction). As compared to the value $1.2 \mathrm{fm}$, known from conventional nuclear physics, the quark gas nuclei reach a rather significant compression. This compression is acted against by the Coulomb force, i.e., $r_{0}(A)$ becomes greater when $(8)$ is included (dashed line). Finally, the full line was obtained after the finite mass of the down quark was incorporated too. This is easily done by setting

$$
\mu(A)=m_{d} R=m_{d} R_{0}(A)=m_{d} A^{1 / 3} r_{0}
$$

for the dimensionless mass, and performing the analysis as indicated above [the value of $r_{0}$ was chosen to be consistent with the result of our calculation in the asymptotic limit for large $A$; for $B=(145 \mathrm{MeV})^{4}$ we find $r_{0}=0.989$

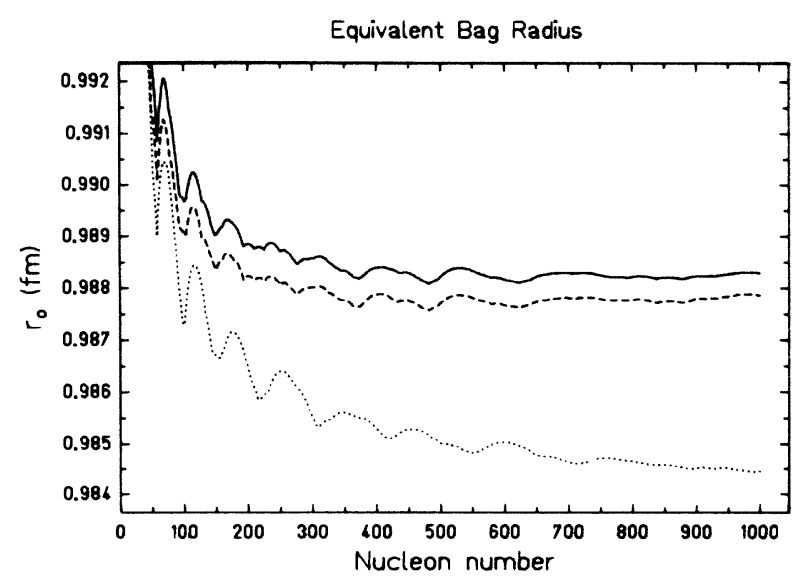

FIG. 1. The equivalent radius of the GQN as a function of $A$. Obviously these objects are significantly compressed as compared with the ordinary nuclear matter ( $r_{0}$ of the order $1.1-1.2$ $\mathrm{fm})$. The compression is smaller, however, when the action of the Coulomb force is allowed for (dashed line) and the downquark mass is finite (full line). The structure of the curves reflects the shell effects.

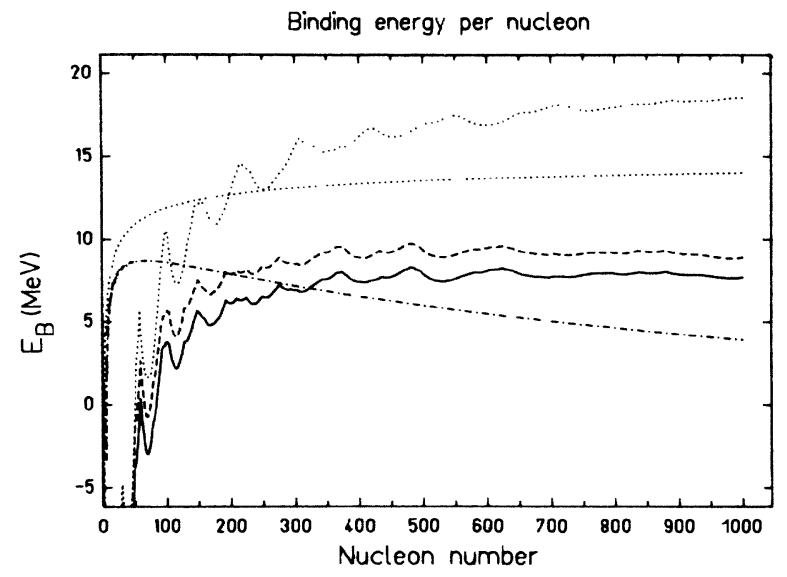

FIG. 2. Binding energy per nucleon as a function of $A$. If the Coulomb energy is excluded, the binding energy for giant quark nuclei (dotted curve) exceeds that of ordinary nuclear matter, described by the Bethe-Weizsäcker formula without the Coulomb term (dotted smooth line), in the uranium region. The crossing point shifts, if the Coulomb energy is included in both formulas (Bethe-Weizsäcker without pairing, dash-dotted smooth line, and our bag calculation, dashed curve) towards heavier nuclei. The shift is amplified if the mass of the down quark is nonzero $\left(m_{d}=5 \mathrm{MeV}\right.$, full curve). In all cases the quarks do not interact via gluon exchange in the bag calculations.

fm]. This obviously allows us to avoid complications in the minimization procedure due to the $R$ dependence of $\mu$ and is a good approximation, although not rigorously correct.

To illustrate the relative contribution of the shell structure (symmetry energy), the Coulomb energy, and the finite quark mass, the binding energies per nucleon

$$
E_{B}(A) \equiv-\left[M_{\mathrm{tot}}(A) / A-m^{\exp }\right]
$$

with

$$
m^{\text {exp }}=938.3 \mathrm{MeV}
$$

are displayed in Fig. 2. In formula (26) we have subtracted the experimentally determined proton mass (note that the average nucleon mass is $\sim 0.4 \mathrm{MeV}$ higher) and not the "consistent" mass of the $A=1$ bag with three free quarks, which without the hyperfine splitting due to onegluon exchange corresponds (up to zero-point energy) to the average mass of the nucleon-delta multiplet ${ }^{3}$

$$
m^{\text {cons }}=M_{\text {tot }}(A=1) \simeq 1217 \mathrm{MeV},
$$

because we wish to compare GQN with free protons for which we know that the noninteracting quark model is not applicable. Note that the relation

$$
M_{\mathrm{tot}}(A)=\frac{16 \pi}{3} R^{3}(A)
$$

holds rigorously in the MIT bag model with free quarks. 


\section{PROPERTIES OF GIANT QUARK NUCLEI}

Let us first list the assumptions hidden in our approximation once more and discuss then their consequences for the properties of GQN. Basic to our considerations is the applicability of the MIT bag model. This approach differs from other bag models in the way the surface of the confining cavity is treated and the chiral symmetry with its associated pion field is taken care of. ${ }^{21}$ The discontinuous surface used in the MIT approach leads to severe conceptional difficulties ${ }^{2}$ (for example the contradiction between the finite volume energy within and the infinite quark mass outside the bubble), but allows, on the other hand, for substantial simplification of the calculational effort with good "first order" results in explaining hadron spectroscopy. ${ }^{19} \mathrm{~A}$ model in which the surface is treated properly in the form of a scalar field coupled to the fermion field is the soliton bag model. ${ }^{4}$ We expect, however, that with increasing number of quarks the inhomogeneity in the scalar field, i.e., the "hole" in the vacuum condensate dug by the quarks, will simply grow in its spatial extension, not changing the properties of the bag walls, such that the contribution of the surface energy will decrease in relation to the volume energy, thus reaching the MIT limit for very large baryon numbers. However, in Fig. 2 we can see a significant "surface" contribution to binding energy, in the sense that an expansion of the binding energy per particle in powers of $A^{-1 / 3}$ gives, to first order, for the dotted curve (no Coulomb energy) with the shell effects smoothed out

$$
E_{B}(A)=a_{1}^{Q}-a \frac{Q}{2} A^{-1 / 3}
$$

with a large value of the "surface parameter" $a_{2}^{0}$ of the order $90 \mathrm{MeV}$ for GQN, whereas for ordinary nuclei we know $^{24} a_{S} \sim 20 \mathrm{MeV}$ (see below). This is to be understood as a parametric expression for the different slopes of the corresponding curves in the region of moderate baryon numbers, and does not mean that the surface energy is necessarily large. Although $a \frac{Q}{2}$ may change if the surface is treated self-consistently and all residual interactions are properly included, the shift still might leave the relation

$$
a_{2}^{Q}>a_{S}
$$

unchanged. This opens, at least in principle, the possibility for hadronic matter to exist in the nucleonic phase for small baryon numbers, when the contribution of the "surface" term is important, and in the quark gas phase for large baryon numbers, when this term becomes negligible. The relation of the volume parameters $a \& \propto B^{1 / 4}$ and $a_{V}$ (see below) tells us, on the other hand, whether the quark phase will be reached at all, i.e., whether asymptotically the binding energy of GQN is greater or smaller than that of ordinary nuclei. In order to "prevent" known matter from being in the quark phase, i.e., to locate the crossing between the dotted curves above $A \sim 250$, we find

$$
B^{1 / 4} \geq 1.00037 \times 145 \mathrm{MeV} \text {. }
$$

On the other hand, if GQN should exist at all for some $A>250$, we find

$$
B^{1 / 4}<1.012 \times 145 \mathrm{MeV} \text {, }
$$

and are left with only a small window of $1.5 \mathrm{MeV}$ above the value of $B^{1 / 4}$ employed in our calculation, for which the existence of GQN is possible. Note that shell effects can change this result only insignificantly. This, however, does not necessarily mean that the discussion about the existence of giant quark nuclei is based on a terrible accident, and that GQN will, with a probability very close to one, not be found in nature. The reason is that the vacuum pressure used in our calculation can be regarded as representing an effective quantity, in which the effects of interactions are absorbed. If the residual color interactions are state dependent, as we expect them to be, and if there are configurations (color singlets) in which they are attractive, the effective value of $B^{1 / 4}$ may, with increasing $A$, cross the border of $145 \mathrm{MeV}$ from above, in particular if the onset of color-conductivity is governed by a scale parameter ("correlation length") different from the hadronic scale. Even if we do not find the nuclear quark phase in laboratory experiments, it could exist in macroscopic cosmic objects like neutron stars.

Now we turn towards the physical properties of giant quark nuclei. First we shall look at the quark density distribution in the bag which is given by

$$
\rho_{i}(r)=\sum \psi_{i}^{+} \psi_{i} \quad i=u, d
$$

where the sum runs over all occupied modes for up ( $i=u$ ) and down $(i=d)$ quarks, and

$\Psi_{i}^{n \kappa v}(\mathbf{r})=A_{\kappa n}\left(\begin{array}{c}j_{l_{\kappa}}\left(\frac{\omega_{\kappa n} r}{R_{0}}\right) \chi_{\kappa}^{\nu}(\Omega) \\ -\frac{i \omega_{\kappa n}}{\epsilon_{\kappa n}+\mu} \operatorname{sgn}(\kappa) j_{\kappa}\left(\frac{\omega_{\kappa n} r}{R_{0}}\right) \chi_{-\kappa}^{\nu}(\Omega)\end{array}\right)$

is the quark wave function (note that $\mu=0$ and $\epsilon=\omega$ for up quarks) with the angular momentum

$$
l_{\kappa}=\left\{\begin{array}{lr}
\kappa & \text { for } \kappa>0 \\
-\kappa-1 & \text { for } \kappa<0
\end{array}, \bar{l}_{\kappa}=l_{-\kappa},\right.
$$

and the magnetic quantum number

$$
v=-j,-j+1, \ldots, j \text {, }
$$

connected with the total spin

$$
j=|\kappa|-\frac{1}{2}
$$

and the two component spherical spinors ${ }^{22}$

$\chi_{\kappa}^{v}(\Omega)=\sum_{m} \sqrt{2 j+1}\left(\begin{array}{ccc}l & \frac{1}{2} & j \\ v-m & m & -v\end{array}\right) Y_{l}^{v-m}(\Omega) \chi^{m}$,

with

$$
\chi^{1 / 2}=\left(\begin{array}{l}
1 \\
0
\end{array}\right), \chi^{-1 / 2}=\left(\begin{array}{l}
0 \\
1
\end{array}\right)
$$

The normalization factor is

$$
A_{\kappa n}=\frac{\left|\omega_{\kappa n}\right|}{\left|j_{l_{\kappa}}\left(\omega_{\kappa n}\right)\right| R_{0}^{3 / 2}}\left[2 \epsilon_{\kappa n}\left(\kappa+\epsilon_{\kappa n}\right)+\mu\right]^{-1 / 2},
$$


where $\epsilon_{\kappa n}$ denotes the energy eigenvalue (1d) corresponding to the eigenspinors (30).

For closed shells with a specific value of $\kappa$ we sum over all magnetic quantum numbers, obtaining

$$
\sum_{v}\left(\Psi^{n \kappa v}\right)^{\dagger} \Psi^{n \kappa v}=\left|A_{\kappa n}\right|^{2}\left[j_{l_{\kappa}}^{2}+j_{\kappa}^{2}\right] \sum_{v}\left(\chi_{\kappa}^{v}\right)^{\dagger} \chi_{\kappa}^{v} .
$$

The sum can be evaluated by inserting the definition(34) and profiting from the properties of the $3 j$ symbols: $:^{23}$

$$
\begin{aligned}
\sum_{v}\left(\chi_{\kappa}^{v}\right)^{\dagger} \chi_{\kappa}^{v} & =(2 j+1) \sum_{v} \frac{1}{2 l_{\kappa}+1}\left|Y_{l_{\kappa}}^{v}\right|^{2} \\
& =\frac{2 j+1}{4 \pi}=\frac{2|\kappa|}{4 \pi} .
\end{aligned}
$$

As expected, closed shells are rotationally invariant. For open shells this will not be the case; such quark nuclei can possibly deform, as do ordinary open shell nuclei. We neglect, however, the deformation of the bag also in this case, and replace the total degeneracy $2|\kappa|$ in (38) by the number which corresponds to the actually occupied states in the open shell. The result is

$\rho_{i}(r)=\sum_{\kappa n} \frac{1}{4 \pi} D(\kappa)\left|A_{\kappa n}\right|^{2}\left[j_{l_{\kappa}}^{2}\left(\frac{\omega_{\kappa n} r}{R_{0}}\right)+j_{\kappa}^{2}\left(\frac{\omega_{\kappa n} r}{R_{0}}\right)\right]$,

with $n$ and $\kappa$ running now over occupied shells and $D(\kappa)$ being the corresponding quark number [i.e., $D(\kappa)=6|\kappa|$ for each isospin direction, if the shell is closed]. Having calculated the expressions (39) for up and down quarks, the baryon density can be written

$$
\rho_{B}=\frac{1}{3}\left(\rho_{u}+\rho_{d}\right)
$$

and the charge density is

$$
\rho_{C}=\frac{2}{3} \rho_{u}-\frac{1}{3} \rho_{d} \text {. }
$$

In Fig. 3 the baryon and the charge densities for double

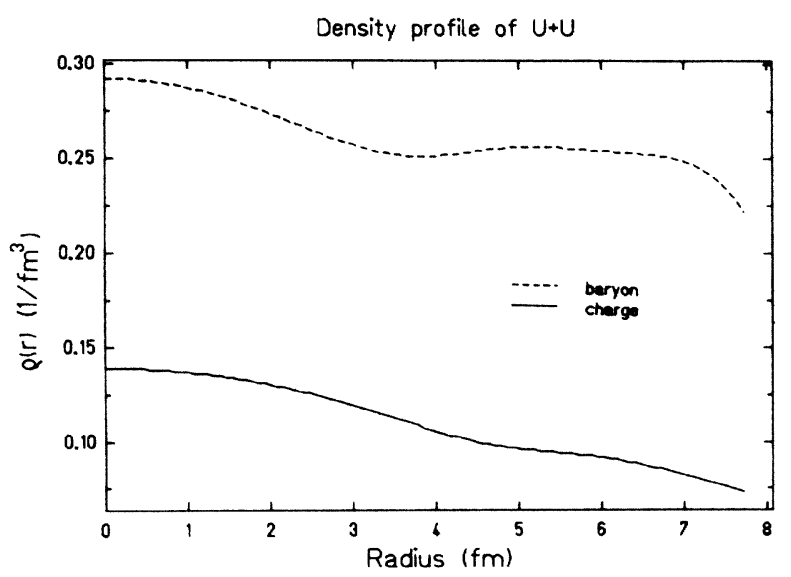

FIG. 3. Density of double-uranium ( $A=476, Z=184$ ). The dotted line is the baryon, and the full line the charge density distribution. This giant quark nucleus has a radius of $7.8 \mathrm{fm}$ which should be contrasted with the radius of a conventional giant nucleus made of nucleons and bound to the standard law $R=1.2 \mathrm{fm} \mathrm{A}^{1 / 3}$, i.e., $R_{\mathrm{UU}}=9.4 \mathrm{fm}$. uranium are plotted as the physical quantities of interest [recall that from (40) and (41) $\rho_{u}=\rho_{B}+\rho_{C}$ and $\left.\rho_{d}=2 \rho_{B}-\rho_{C}\right]$. On the average we indeed find these nuclei more or less homogeneous. However, the repulsion of the charge distribution towards the surface (polarization of GQN) is not contained in this treatment, since the energy levels are not allowed to rearrange due to the Coulomb force, and a depletion of levels with low angular momentum (wave function concentrated in the center of the bag) in favor of high angular momentum states (wave function concentrated towards the bag walls) does not occur in this zeroth order estimate. Obviously, a self-consistent (Hartree-Fock) calculation would be required to take the polarization effects due to the Coulomb and eventually also color forces into account. Clearly, much work is still to be done before reliable conclusions can be drawn.

In spite of this we shall try to glance at the physical implications of our model, and return to Fig. 2 again. The Coulomb energy and the finite quark mass are not included in the upper (dotted) line displaying the binding energy per nucleon (i.e., per three quarks) which crosses the binding energies per nucleon obtained by extrapolating the empirically determined Bethe-Weizsäcker formula supposed to represent ordinary nuclear matter. The latter is given by ${ }^{24}$

$$
\begin{aligned}
E_{B}(A, Z)= & a_{V}-a_{S} A^{-1 / 3}-a_{C} Z^{2} A^{-4 / 3} \\
& -a_{A}\left[\left(Z-\frac{1}{2} A\right) / A\right]^{2}+a_{P} A^{-3 / 2} \delta,
\end{aligned}
$$

with the parameters

$$
\begin{aligned}
& a_{V}=15.85 \mathrm{MeV}, \\
& a_{S}=18.34 \mathrm{MeV}, \\
& a_{C}=0.71 \mathrm{MeV}, \\
& a_{A}=92.86 \mathrm{MeV}, \\
& a_{P}=11.46 \mathrm{MeV}, \\
& \delta=\left\{\begin{array}{l}
+1 \text { for even-even nuclei } \\
-1 \text { for odd-odd nuclei } \\
0 \text { otherwise }
\end{array}\right.
\end{aligned}
$$

fitted to experimental data. The smooth dotted curve represents the Bethe-Weizsäcker formula with the Coulomb energy omitted. It crosses the analogous curve for quark nuclei in the region of uranium. In this case the $Z / A$ ratio is $\frac{1}{2}$ on average due to the action of the Pauli principle. If we include the Coulomb interaction in the schematic way discussed previously, the binding energy (dashed curve) and the $Z / A$ ratio decrease. The BetheWeizsäcker formula with the Coulomb term included now, yields binding energies indicated by the smooth dash-dotted line. The crossing between the two curves shifts, however, only slightly. A much larger shift of the crossing point into the region of giant elements arises if the finite mass of the down quark (lower full line) is included in the determination of the masses of GQN. Note that on the average the $Z / A$ ratios obtained from the minimization of the Bethe-Weizsäcker formula (which is 


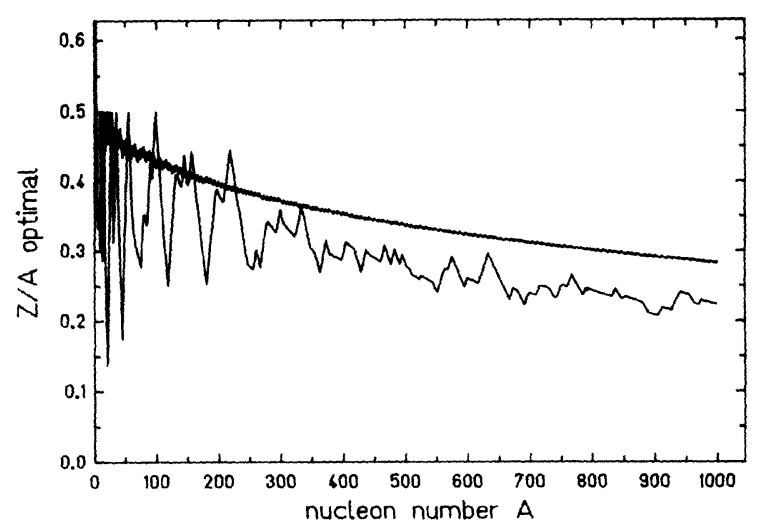

FIG. 4. The $Z / A$ ratio: It is obtained by minimalization of the binding energy with respect to the total charge $Z$ with $A$ held constant. The results of our bag calculation (full line) differ significantly from those obtained in an independent minimization of the Bethe-Weizsäcker binding energy (dashed line) for high baryon numbers.

based on the liquid-drop picture for the atomic nucleus and does therefore not contain shell corrections) differ from those obtained in our bag calculation (see Fig. 4) significantly in the region of giant nuclei. Giant quark nuclei tend, at least in our approximation, to large "neutron" excess. It is only the stable valleys $\left[\boldsymbol{Z}=\boldsymbol{Z}_{\mathrm{st}}(\boldsymbol{A})\right]$ for nucleonic and quark nuclei which are exhibited in Fig. 4. The "neutralization" of a GQN with $Z>Z_{\text {st }}$ charges can proceed either via proton or $\alpha$-particle emission or via beta decay. Fission of GQN seems to be energetically less favorable than in the case of regular nuclei (cf. Fig. 2); it should, however, be possible. All these decay modes of GQN deserve further investigation, which we shall not carry out here. Note that relativistic electrons will also be present inside the highly charged quark nuclei and will contribute to their stability.

The consequences of our model are now the following: If the effective bag pressure happens to be $(145 \mathrm{MeV})^{4}$ or below for some baryon number $A$, the nucleons within such a giant nucleus dissolve into quarks. This transition is expected to be very fast, i.e., on the typical nuclear time scale $\tau \lesssim 10^{-21} \mathrm{sec}$. Again, the study of its time development is an important task for the future. The shell structure in GQN is different from the shell structure in ordinary nuclei, as far as the noninteracting model is considered. As already noticed some time ago (see Bleuler et al., Ref. 12), the use of the Dirac equation (1) automatically generates the right ordering of angular momenta which in the nonrelativistic model has to be enforced by including the spin-orbit coupling. ${ }^{25}$ In the MIT bag model the magic numbers are $2,6,8,14,(18,20), 28,34, \ldots$, as compared with the conventional $2,(6), 8,(14,16), 20,28,(38,40), \ldots$ (in parentheses the minor shell closures are indicated).

\section{POSSIBLE EXPERIMENTAL CONSEQUENCES}

It is clear that the anomalous objects discussed here can be found in an experimentally accessible range of baryon numbers only if the interactions between the liberated quarks are attractive and strong enough to force the effective bag constant below $145 \mathrm{MeV}$, but only for baryon numbers greater than 250 and smaller than those available in collisions between very heavy ions. But even if this is the case, we have to realize that the search for anomalous states of nuclear matter has been put forward in highenergy heavy-ion collisions in which, due to the existence of nuclear shock waves, ${ }^{26}$ high-temperature and highdensity phases can be reached, but where we are limited to small projectiles. Effects of large baryon numbers can therefore be seen only with machines which are able to accelerate projectiles up to the heaviest ones. Since such devices like the UNILAC at GSI are, on the other hand, yet limited with respect to the ion final velocity, nobody has looked for such effects there, particularly because of the common expectation that quark structure is restricted to showing up in the very high energy domain.

As already mentioned above, an anomalous positron production has been measured in recently reported experiments with heavy ions accelerated up to velocities in the vicinity of the Coulomb barrier. These experiments were carried out over nearly a decade to study the expected instability of the quantum electrodynamics (QED) vacuum in very strong ("supercritical") electric fields generated by giant nuclear systems. ${ }^{14}$ For long-lived such systems $\left(\tau \geq 3 \times 10^{-20} \mathrm{sec}\right)$ a sharp resonance should appear in the positron spectrum, when the overcritical charge of the united system is partially screened by electrons created out of the vacuum, and the total charge is balanced by positron emission. Such positron lines have indeed been found in various experiments, ${ }^{27}$ but its dependence on the positron kinetic energy seems not to show the scaling with $Z=Z_{\text {proj }}+Z_{\text {target }}$, expected on the grounds of standard extrapolation of the properties of ordinary nuclear matter to the domain of giant nuclei. The position of the "spontaneous" peak in the positron spectrum is rather energy independent at $T_{\text {pos }} \sim 320 \mathrm{keV}$.

What happens now, if we suppose that giant quark nuclei are created in these experiments? Can the existence of a GQN account for these observations? Of course, the nuclear physics, which so sensitively determines the above-mentioned scaling behavior of the positron resonance energy, is dramatically changed. However, in trying to answer this question we have to be careful, since

TABLE II. Baryon and charge numbers of several realistic "giant" collision systems presently under investigation at GSI.

\begin{tabular}{lcc}
\hline \hline System & $A$ & $Z$ \\
\hline $\mathrm{Pb}+\mathrm{Pb}$ & 416 & 164 \\
$\mathrm{Ta}+\mathrm{U}$ & 419 & 165 \\
$\mathrm{Au}+\mathrm{U}$ & 435 & 171 \\
$\mathrm{~Pb}+\mathrm{U}$ & 446 & 174 \\
$\mathrm{~Pb}+\mathrm{Cm}$ & 455 & 178 \\
$\mathrm{Th}+\mathrm{Th}$ & 464 & 180 \\
$\mathrm{Th}+\mathrm{U}$ & 470 & 182 \\
$\mathrm{U}+\mathrm{U}$ & 476 & 184 \\
$\mathrm{Cm}+\mathrm{Th}$ & 479 & 186 \\
$\mathrm{Cm}+\mathrm{U}$ & 485 & 188 \\
\hline \hline
\end{tabular}


TABLE III. The binding energies $\left(E_{B}\right)$ of GQN, equally sized normal nucleus (EBW), and the gain in binding energy $\left(\Delta E_{B}\right)$ after transition into the quark gas state. The radius of the GQN as well as the positron kinetic energies are also given.

\begin{tabular}{lccccr}
\hline \hline System & $\begin{array}{c}E_{B} \\
(\mathrm{MeV})\end{array}$ & $\begin{array}{c}\text { EBW } \\
(\mathrm{MeV})\end{array}$ & $\begin{array}{c}\Delta E_{B} \\
(\mathrm{MeV})\end{array}$ & $\begin{array}{c}R \\
(\mathbf{f m})\end{array}$ & $\begin{array}{c}T_{\text {pos }} \\
(\mathrm{keV})\end{array}$ \\
\hline $\mathrm{Pb}+\mathrm{Pb}$ & 5.89 & 6.16 & -0.27 & 7.38 & \\
$\mathrm{Ta}+\mathrm{U}$ & 5.88 & 6.14 & -0.26 & 7.39 & \\
$\mathrm{Au}+\mathrm{U}$ & 5.98 & 6.03 & -0.05 & 7.49 & 22.48 \\
$\mathrm{~Pb}+\mathrm{U}$ & 6.13 & 5.98 & +0.15 & 7.55 & 130.65 \\
$\mathrm{~Pb}+\mathrm{Cm}$ & 6.16 & 5.89 & +0.27 & 7.60 & 291.81 \\
$\mathrm{Th}+\mathrm{Th}$ & 6.23 & 5.87 & +0.36 & 7.65 & 376.66 \\
$\mathrm{Th}+\mathrm{U}$ & 6.10 & 5.83 & +0.27 & 7.69 & 467.73 \\
$\mathrm{U}+\mathrm{U}$ & 6.02 & 5.79 & +0.23 & 7.72 & 562.10 \\
$\mathrm{Cm}+\mathrm{Th}$ & 5.96 & 5.75 & +0.21 & 7.73 & 663.31 \\
$\mathrm{Cm}+\mathrm{U}$ & 5.93 & 5.71 & +0.22 & 7.77 & 767.78 \\
\hline \hline
\end{tabular}

the reaction systems with high baryon numbers used in actual experiments do not have a total charge which coincides with the minimum charge $Z$ as calculated in Sec. II. Their charge is rather fixed by the incoming more or less stable nuclei (e.g., $\mathrm{U}+\mathrm{U}, \mathrm{U}+\mathrm{Cm}, \mathrm{U}+\mathrm{Th}, \mathrm{Th}+\mathrm{Th}$, etc.), and since the expected lifetime of the unified system is too short for weak processes, it remains unchanged (up to a possible emission of charged light clusters ${ }^{28}$ ) during the reaction.

In Table II the systems which have been or will be used in actual GSI experiments are listed. The binding energies of these systems are not contained in Fig. 2, where only the nuclei along the valley of stability ( $Z$ minimized for a given $A)$ are displayed. And, since in the BetheWeizsäcker formula (without shell corrections) the charge has also been minimized to obtain the binding energy of ordinary nuclear matter in Fig. 2, we also have to compare our results for the combined systems with the binding energies of normal nuclei with the fixed charges, off

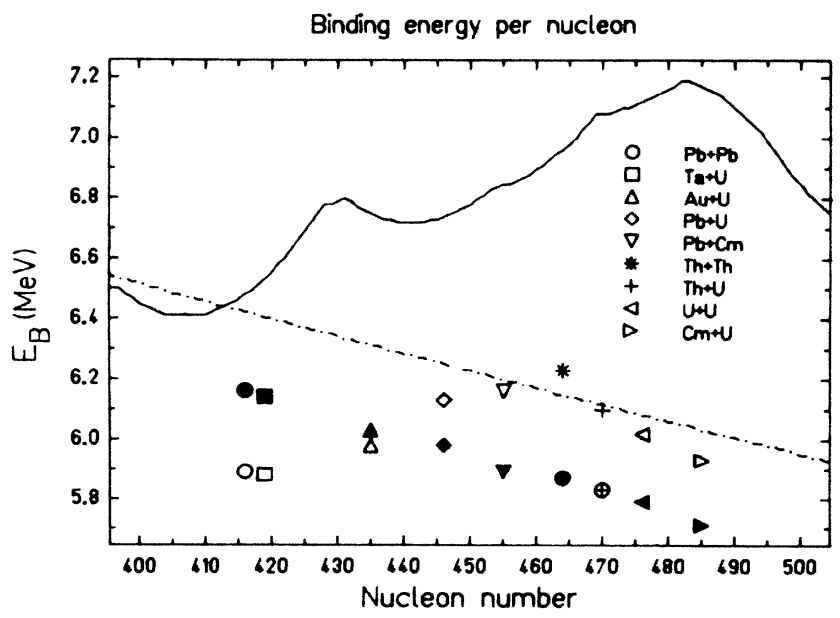

FIG. 5. Binding energy of realistic giant quark systems. The open symbols are the GQN, the solid ones the corresponding normal nuclei (Bethe-Weizsäcker formula). The solid line is the one already displayed in Fig. 2; the dashed-dotted line is BetheWeizsäcker matter. the corresponding stable valley. This is listed in Table III and shown also in Fig. 5. Note first that for $B^{1 / 4}=145.00 \mathrm{MeV}$ the difference in binding energy per nucleon for quark nuclei and regular nuclei becomes small in the region of baryon numbers between 400 and 500; in this calculation, where shell corrections for the liquid drop theory are omitted, ordinary nuclei seem to be favored for systems like $\mathrm{Pb}+\mathrm{Pb}, \mathrm{Ta}+\mathrm{U}$, but quark nuclei favored particularly for $\mathrm{Th}+\mathrm{Th}$ and its neighbors. This is a consequence of the shell structure: For $\mathbf{T h}+\mathrm{Th}$ the number of up quarks is 644 , the number of down quarks 748 [cf. Table II and Eq. (5)], both just above the respective "magic" numbers $(642)\left(4 s_{1 / 2}\right.$ shell filled) and 744 (first $\kappa=8$ shell filled). The energy gain of $\sim 0.36$ $\mathrm{MeV} /$ nucleon relative to the normal nuclear matter creates a "pocket" in the interion potential with the depth given by $2 \times A_{\mathrm{Th}} \times 0.36 \mathrm{MeV} \simeq 167 \mathrm{MeV}$, and could be a new explanation of the long lifetime of the unified system, sufficient to destroy any vacancy in the electronic $1 s$ level by spontaneous positron production. The arising decrease of the nucleus-nucleus potential at the spherical point is even, although a little too large, of the correct order of magnitude required to reach a very stable nuclear structure of these giant objects. ${ }^{14}$ Moreover, since $\mathrm{Th}+\mathrm{Th}$ seems to be the combination with the tightest binding and hence with the possibly longest lifetime, it is not impossible that all spontaneous positrons are emitted from this system, even though the reaction systems are heavier. The missing mass is perhaps emitted in the form of small yet undetected fragments.

\section{OUTLOOK}

If the positrons are always emitted from the same giant unified system, there is no miracle concerning their constant energy. On the other hand, the quark-gas $\mathrm{Th}+\mathrm{Th}$ system has a radius of $\sim 7.66 \mathrm{fm}$, i.e., it is significantly smaller (similarly, the other GQN are very much more compressed than their "brothers" made of nucleons) than the conventional unified $\mathrm{Th}+\mathrm{Th}$ spherical giant system (radius of $\sim 9.2 \mathrm{fm}$ ). Since the positron kinetic energy depends very sensitively upon the radius of the charge distri- 
bution, then, if taken seriously, the anomalous positron peak should ${ }^{29}$ be centered around $\sim 0.4 \mathrm{MeV}$ in this case. This is, however, far above the experimentally found value of $320 \mathrm{keV}$. This fact, as well as the too large binding energy mentioned in the preceding section, indicates that we have perhaps overestimated the compression of GQN. A more careful theoretical analysis of the Coulomb and color corrections might improve on this point, though.

If the assertion that the positrons are always emitted from the same giant system seems unacceptable, then the position of the positron peak will, of course, depend on the collision system under consideration. Discarding for a moment all doubts we still have about the correctness of our results in view of their sensitivity to the choice of the model parameters and of the crude approximations made, the positron energies can easily be calculated. ${ }^{29}$ The result is listed in Table III.

It also should be mentioned that since GQN are supposed to be highly polarizable, in particular by the action of the long range electromagnetic force, the arising strong electric and magnetic fields may, depending on the geometry of the system, induce significant enhancements of various types of fermionic (scalar, pseudoscalar, etc.) densities which in turn could serve as anomalous sources for nonperturbative creation of new particles. Such particles, if decaying into the electron-positron channel, could eventually also produce sharp monoenergetic positron resonances. $^{30}$

\section{ACKNOWLEDGMENTS}

We thank A. Schäfer, P. Schlüter, T. deReus, and H.-T. Elze for valuable discussions and $W$. Swiatecki for stimulating comments, particularly on the significance of the "surface" term, and for careful reading of the manuscript. This work was supported by GSI Darmstadt, the Bundesministerium für Forschung und Technologie, Bonn, and by the U.S. Department of Energy under the Contract DE-AC03-76SF00098. D.V. gratefully acknowledges a DAAD-NATO Postdoctoral Fellowship.
${ }^{1}$ For a review, see, e.g., K. Johnson, Acta Phys. Pol. B 6, 865 (1975); P. Hasenfratz and J. Kuti, Phys. Rep. 40, 75 (1978); A. W. Thomas, Adv. Nucl. Phys. 13, 1 (1983).

${ }^{2}$ D. Vasak, Gesellschaft für Schwerionenforschung Report 85$12,1985$.

${ }^{3}$ A. Chodos, R. L. Jaffe, K. Johnson, C. B. Thorn, and V. F. Weiskopf, Phys. Rev. D 9, 3471 (1974); A. Chodos, R. L. Jaffe, K. Johnson, and C. B. Thorn, ibid. 10, 2599 (1974).

${ }^{4}$ R. Friedberg and T. D. Lee, Phys. Rev. D 15, 1694 (1977); 16, 1096 (1977); R. Friedberg and T. D. Lee, ibid. 18, 2623 (1978); R. Goldflam and L. Wilets, ibid. 25, 1951 (1982); R. Goldflam and L. Wilets, Comments Nucl. Part. Phys. 12, 191 (1984).

${ }^{5}$ R. L. Jaffe, Title, Proceedings of the International School of Physics, "Ettore Majorana," Erice, Italy, 1979, edited by editors (Publisher, City, 19xx); G. E. Brown and M. Rho, Phys. Lett. 82B, 177 (1979).

6S. Theberge, A. W. Thomas, and G. A. Miller, Phys. Rev. D 22, 2838 (1980); S. Theberge and A. W. Thomas, Nucl. Phys. A393, 252 (1983).

${ }^{7}$ V. Noble, Phys. Rev. Lett. 46, 412 (1981); J. Aubert, Phys. Lett. 123B, 123 (1983); 123B, 275 (1983); A. Bodek, Phys. Rev. Lett. 50, 1431 (1983); 51, 534 (1983).

${ }^{8}$ M. Betz, G. Krein, and T. A. J. Maris, Nucl. Phys. A437, 509 (1985); K. Bräuer, A. Fässler, and K. Wildemuth, ibid. A434, 717 (1985); for a review see K. Rith, University Freiburg Report THEP 83/4, 1983.

${ }^{9}$ F. E. Close, R. G. Roberts, and G. G. Ross, Phys. Lett. 129B, 346 (1983); O. Nachtmann and H.-J. Pirner, Z. Phys. C 21, 277 (1984).

${ }^{10}$ B. Banerjee, N. K. Glendenning, and V. Soni, Phys. Lett. 155B, 213 (1985).

${ }^{11}$ C. Derreth, A. Schäfer, Q. Zhang, and W. Greiner, J. Phys. G 12, L19 (1986).

${ }^{12}$ We cannot resist noting here that the breakdown of the scalar field in the interior of the nucleus is of the same kind as that expected in the so-called Lee-Wick isomers; see T. D. Lee and G. C. Wick, Phys. Rev. D 9, 2291 (1974). Surprisingly enough, the condensation energy density, needed to create a Lee-Wick nucleus, coincides quite well with the vacuum pressure used in bag models, if parameters conventionally used in mean-field theories (see, e.g., P.-G. Reinhard et al., Ref. 17) are chosen. It seems therefore not too remote to consider the GQN as a realization of Lee-Wick nuclei on the quark level. On the other hand, we do not attempt to explain the nature of well-known nuclei on the quark level; there is therefore no overlap of our work with the investigations of the Bonn group; see, e.g., K. Bleuler, H. Hofestädt, S. Merk, and H. R. Petry, Z. Naturforsch. 38a, 705 (1983).

${ }^{13}$ Proceedings of the 3rd International Meeting on Small Particles and Inorganic Clusters, Berlin, Federal Republic of Germany, 1984, edited by K. H. Bennemann and J. Koutecky, Surf. Sci. 156, (1985); J. A. A. J. Perenboom, P. Wyder, and F. Meier, Electronic Properties of Small Metallic Particles (North-Holland, Amsterdam, 1981).

${ }^{14} \mathrm{~W}$. Greiner, in Proceedings of the International Conference on Nuclear Physics, Florence, Italy, 1983, edited by P. Blasi and R. A. Ricci (Tipografia Compositori, Bologna, 1983), p. 635; in Proceedings of the Second International Conference on Nucleus-Nucleus Collisions at Visby, Sweden, 1985; in Proceedings of the Summer School on Nuclear Physics at La Rabida, Spain, 1985, edited by G. Madurga.

${ }^{15}$ M. Seiwert, W. Greiner, and W. T. Pinkston, J. Phys. G 11, L21 (1985).

${ }^{16}$ M. Seiwert, J. A. Maruhn, W. Greiner, and J. Friedrich, Z. Phys. A 321, 653 (1985).

${ }^{17}$ P. G. Reinhard, M. Rufa, J. Maruhn, W. Greiner, and J. Friedrich, Z. Phys. A 323, 13 (1986).

${ }^{18}$ M. Flensburg, C. Peterson, and L. Sköld, Z. Phys. C 22, 293 (1984); T. C. E. Carlson, T. H. Hansson, and C. Petersen, Phys. Rev. D 27, 1556 (1983).

${ }^{19}$ T. DeGrand, R. L. Jaffe, K. Johnson, and J. Kiskis, Phys. Rev. D 12, 2060 (1975).

${ }^{20}$ Note that due to the absence of color interaction the strong hyperfine splitting between nucleons and $\Delta$ resonances is also absent. 
${ }^{21}$ P. J. Mulders and A. W. Thomas, J. Phys. G 9, 1159 (1983); T. Radozycki and S. Tatur, Phys. Rev. D 31, 1074 (1985).

${ }^{22}$ E. M. Rose, Relativistic Electron Theory (Wiley, New York, 1961); W. Greiner, Theoretische Physik VI, Relativistische Quantentheorie, Wellengleichungen (Deutsch, Thun und Frankfurt, 1981).

${ }^{23}$ M. Rotenberg, R. Bivins, N. Metropolis, and J. K. Wooten, Jr., The 3-j and 6-j Symbols (Technology, Cambridge, Mass., 1959).

${ }^{24}$ A. H. Wapstra, Atomic Masses of Nuclides, Vol. 38 of Handbuch der Physik, edited by S. Flügge (Springer, Berlin, 1958).

25J. Eisenberg and W. Greiner, Nuclear Theory (North-Holland, Amsterdam, 1976), Vol. I.

${ }^{26}$ H. Stöcker and W. Greiner, Phys. Rep. 137, 277 (1986).

${ }^{27}$ M. Clemente, E. Berdermann, P. Kienle, H. Tsertos, C. Kozhuharov, F. Bosch, and W. König, Phys. Lett. 137B, 41 (1984); J. Schweppe, A. Gruppe, K. Bethge, H. Bokemeyer, T. Cowan, H. Folger, J. S. Greenberg, H. Grein, S. Ito, R.
Schule, D. Schwalm, K. E. Stiebing, N. Trautmann, P. Vincent, and M. Waldschmidt, Phys. Rev. Lett. 51, 2261 (1983); T. Cowan, H. Backe, M. Begemann, K. Bethge, H. Bokemeyer, H. Folger, J. S. Greenberg, H. Grein, A. Gruppe, Y. Kido, M. Klüver, D. Schwalm, J. Schweppe, K. E. Stiebing, N. Trautmann, and P. Vincent, ibid. 54, 1761 (1985); H. Tsertos, E. Berdermann, F. Bosch, M. Clemente, P. Kienle, W. Koenig, C. Kozhuharov, and W. Wagner, Phys. Lett. 162B, 372 (1985).

${ }^{28}$ Dynamically induced and spontaneous cluster emission out of ordinary (nucleonic) giant nuclear systems is investigated by Martin Greiner and Werner Scheid, University of Giessen.

${ }^{29}$ T. J. deReus et al., J. Phys. B 17, 615 (1984); and private communication. The nuclear distributions are assumed to be homogeneous in these calculations.

${ }^{30}$ A. Schäfer, J. Reinhardt, B. Mueller, and W. Greiner, J. Phys. G 11, L69 (1985); J. Reinhardt, A. Schäfer, B. Müller, and W. Greiner, Phys. Rev. C 33, 194 (1986). 